extraction of the stone. When a stone is too large to be removed through the incision without doing violence to the soft parts, it should be fragmentized with suitable instruments and the fragments extracted through the perineal opening.

The crushing and withdrawal of stones through the perineum has been regarded with an unnecessary degree of fear. It is incomparably safer than forcible extraction of large calculi. That it is a safe resort is attested by numerous instances in which soft calculi crushed in the blades of the forceps have been removed piecemeal from the bladder without any evident ill effects. This has happened in a number of cases in which I have operated, and in many I have intentionally crushed large stones through the perineal wound without in the least increasing the risk of an operation.

If the stone is too hard to be readily crushed, the surgeon should not hesitate to make a suprapubic section, the success of which is greatly enhanced by the thorough drainage through the lower opening.

4. It reduces the death-rate to the minimum.

Possessing, as I think it does, the advantages mentioned, it is my conviction that the mediobilateral operation, properly executed, is almost devoid of danger to life, and that in the absence of organic disease of the kidneys or other vital organ, every patient submitted to it will recover. It is followed by very little if any shock, by reason of the slight injury to important structures. It limits the loss of blood to the minimum, is seldom the cause of surgical fever, and in a majority of cases no unpleasant symptoms ensue. Patients are usually up in a week, and in from twelve to eighteen days, as a rule, the wound is entirely healed. In six cases in my practice the wounds healed by the first intention, no water passing the wound after the first day.

The statistics of medio-bilateral lithotomy have, so far as I know, never been collected and published. The late Prof. S. D. Gross, in the last edition of his valuable work on the urinary organs, did report twenty-two cases followed by complete recovery. At the meeting of the American Medical Association at Chicago, in 1877 , I reported forty-six cases, all successful. Since that time I have operated by the same method on ninety cases, making in all I 36 cases, with three deaths.

The success of the medio-bilateral operation has not been limited to my practice. Dr. J. R. Weist, of Richmond, Ind., has reported to me six cases in his practice with successful results. The late Dr. J. W. Thompson, of Paducah, reported twelve cases, all successful. My son, Prof. C. S. Briggs, of Nashville, has performed the operation in five cases, all successful. Dr. Wm. Wright, of Huntingdon, Tenn., operated on a boy i 2 years of age with success, and Dr. D. M. Evans, of
Union City, Tenn., did the operation on an adult with success, making an aggregate of 183 cases, with three deaths, one in sixty-one.

In conclusion I make the following deductions:

I. No operation is adapted to all cases.

2. Litholapaxy is incomparably superior to lithotrity, and should always be practiced in selected cases.

3. The supra-pubic should be chosen for extraction of very large and hard stones.

4. The medio-bilateral should be chosen in all other cases, because :

$a$. It affords the shortest route to the bladder,

$b$. It divides parts of the least importance.

$c$. It is the most bloodless operation.

$d$. It affords space for extraction of any calculus which can be removed through the perineum.

$c$. It affords the best route for drainage.

$f$. It reduces the death-rate to the minimum,

\section{THE NON-SURGICAL, TREATMENT OF} STRABISMUS CONVERGENS.

Read in the Section of Ophthalmology at the Fortieth Annual Meeting of the American Medical Association, June, 1889 .

BY EDWIN J. GARDINER, M.D., OF CHICAGo, ILL.

The non-surgical treatment of strabismus convergens is not a novelty. The text-books on ophthalmology mention the fact that some cases of strabismus convergens, if taken in time, notably in the periodic phase, are amenable to treatment, but the majority of authors quickly dismiss the subject, and hasten on to an elaborate description of the more popular operative process. There are, however, some exceptions, and among. these, Dr. Landolt's excellent book on "Refraction and Accommodation" is prominent; with the clearness and precision which always characterize his work, he enters minutely into the details of the non-surgical method, pointing out its advantages. and giving rules for its execution. In this book he strongly advocates its careful adoption; and in his paper read before the Ninth International Medical Congress, he again sounded a note of warning against operating on cases of strabismus convergens without previously testing with great care the action of the muscles when the proper relation between accommodation and convergence is reëstablished.

It is to be regretted, however, that Dr. Landolt did not emphasize more how much can be accomplished by restoring the balance between these two functions. If the tendency of the age were toward conservatism, a word would have been sufficient, but the trend is quite in the contrary direction and in this matter, at least, it is time to put on the brakes. The treatment of strabismus has suffered from careless execution in the past, and there is danger that it may again suffer from 
indiscriminate and hasty operations in the present. It is really appalling to witness the assurance with which many tyros in ophthalmic surgery perform tenotomy, without a moment's hesitation as to the future results, and plenty of evidence can be adduced to prove that some who are not tyros, firmly believing that tenotomy is the only reliable method, operate too hastily to be consistent with safety. Why this condition of things should be so prevalent is difficult to explain, unless by the supposition that the former are ignorant of the claims that have been made for the non-operative treatment, and that the latter ignore them, being unwilling to allow sufficient time for this method to accomplish the result. That the result is accomplished in time, Nature, our best teacher, is constantly manifesting, for it is a well known fact that many cases of strabismus convergens are spontaneously corrected as the patient advances in years. If Nature accomplishes this feat when left to her own resources, is it not worth while to investigate her method of operation and profit by her teaching?

It is universally admitted that concomitant strabismus convergens, in hypermetropes, is caused by the excessive tension of accommodation necessary for distinct vision, overstepping the limits of the negative range of relative convergence; or, in other words, by the tension of accommodation exceeding the quota of the positive range of relative accommodation. The balance between these correlative functions being destroyed, over-convergence takes place, clear images are obtained at the expense of binocular vision, and strabismus is established. It should not be forgotten, however, that although the balance between accommodation and convergence is destroyed, their correlation is not abolished, and that it is in obedience to the law of reciprocal action which governs it, that over-convergence supervenes, and is maintained. On the survival of this correlation, the possibility of correction entirely rests.

Nature meets the difficulty by gradually limiting the range of accommodation, thus restricting the patient from excessive tension of accommodation, while it leaves the range of convergence unimpaired. When in the course of time these two functions approach equilibrium, the inciting cause having ceased to be operative the strabismus disappears, if the internus has not become permanently shortened. It will be noticed that the main point, in Nature's process, is the restoration of equilibrium between accommodation and convergence. The non-surgical method, in view of this fact, starts out to restore the balance between these functions, by removing the necessity for excessive accommodation, $i, e_{\text {, }}$, by correcting the error of refraction. This, however, is not usually sufficient. Habitual accommodation persists in spite of optical correction. Mydriatics must be brought into operation, and by this means, the very simple operation and devoid of all danger contracted ciliary muscle is temporarily set at rest. Then, if the internal rectus is not permanently shortened, a sometimes rapid, but generally a gradual relaxation of the muscle takes place, and pari passu the squint is diminished or a rapid amelioration may be anticipated, and in many cases of alternating and in some of the established monocular form, the result is at times surprisingly quick. In the latter class of cases, however, an immediate correction is not to be expected, and when, as it usually happens, the deviating eye is quite amblyopic, something should be done in addition, to improve its vision, bring it into action and thus expedite the correction.

Experience teaches, and this is the point, that sooner or later the squint is corrected in a large percentage of the cases. Of course there are some cases in which the internal rectus is hopelessly shortened; in these tenotomy is the only remedy, but it must be first ascertained whether the internus is thus shortened, and this cannot be determined by correcting the ametropia and instilling atropin for a week. And now we have reached the point, where $I$ will be found at issue with many of my professional brethren. A great many ophthalmologists claim that after having properly corrected the ametropia, instillations of atropin for a week will suffice to effect all the change in the degree of convergence that can be expected; in other words, that the muscle in this time has reached the limit of extensibility, and, in accordance with this belief, they proceed to operate at the expiration of this period, and before in many instances. The claim is erroneous, for I have repeatedly witnessed a gradual decrease in the angle of convergence, extending over weeks and months, and although it must be admitted that a case which has not been at all affected by atropin in a week, does not give great promise. the treatment must not be considered a failure until three or four weeks have elapsed. If gradual improvement is noted during the first week, and by careful examination the least amelioration is detected, the treatment is to be continued, even though it may take a long time, in the hope of securing a perfect correction and possibly a cure, without injuring the integrity of the muscle.

It may appear from these statements that I am waging war against tenotomy. Such is not my purpose. In certain cases, nothing can be substituted for a carefully performed tenotomy. But I certainly do protest against hasty operative interference when Nature, properly assisted by science, can and does accomplish the result. I am pleading for the internal recti muscles. Tenotomy needs no champion. But the interni most certainly need a protector.

The assertion that tenotomy of the internal rectus in concomitant strabismus convergens is a corrected. In cases of periodic strabismus quite 
is, to say the least, as misleading as it is common. The technique of the operation is indeed simple, but the delicate discrimination requisite to decide, first, whether an operation is necessary, then what operation is indicated and, finally, how much of an operation to perform, requires an amount of judgment which at once places this operation beyond the pale of extreme simplicity. The second clause of the assertion is absolutely untrue. For if tenotomy is performed without previously having given the non-surgical method a thorough trial, the number of cases in which this method succeeds, will indicate the number which the operation over-corrects. In other words, if in a given case the angle of deviation be equal to $40^{\circ}$ and it can be reduced without operative interference to $10^{\circ}$, and the operation is performed without giving this method a thorough trial, then all that goes to correct over $10^{\circ}$ is over-correction, and in the course of time, an insufficiency of the interni and possibly a divergent strabismus will supervene.

The principal objection raised to this method is that tenotomy does in a few days what it takes several weeks and even months for the other method to accomplish. Now, in the first place tenotomy, if carefully performed, does not accomplish the result in so short a time; and in the second place, it should be borne in mind that the object of every physician is to do that which is ultimately best for the patient. If a case of strabismus convergens is operated before a thorough course of treatment has been carried out, it is simply trusting to chance whether the eyes are to remain parallel, or in the course of time suffer from insufficiency of the interni, or exhibit divergent strabismus. Time, which plays so important a part in this objection, sinks into insignificance and a matter of no consideration, when compared to the possible future discomfort of the patient. This objection certainly loses strength when an. alyzed.

The second objection is that while the method may be true in theory, it is impracticable in its application, and when put to the clinical test results in failure in the majority of cases, This being a practical objection, a practical answer is desirable, and this is best done by the recital of facts, thus allowing these to speak for themselves.

The danger of hasty operative interference was forcibly brought to my notice about nine years ago, when after having used atropin for five or six days, I operated on a very marked case of established strabismus convergens. I was in the habit of allowing the atropin more time to do its work, but there was so much deviation in this instance, that I considered the operation perfectly safe. The result immediately after the operation was very satisfactory, although slight convergence persisted, but about five months afterward the child was brought back to me with a very perceptable divergent squint, and the mother expressed herself very strongly on tha subject of operating on eyes and making them go "the other way." From that date I have had no more cases of operative divergent strabismus, of $\mathrm{my}$ own manufacture, because I have never operated upon a case of strabismus until I had become perfectly satisfied that the non-surgical treatment was insufficient. My experience with this method has been uniformiy gratifying, both as a curative process and as a preliminary process to the operative treatment, and I have always strongly advocated its use both in the lecture room and in the clinic. The patients were always found perfectly willing to undergo the course of treatment, and in many instances a decided preference in its favor was expressed. The discomforts accompanying the use of the mydriatic, when complained of, were much mitigated by the use of stronger glasses for near work, and the orthoptic measures; exercises for the amblyopic eye, etc., were quite well followed in the majority of cases. The latter, however, it must be admitted is the most difficult part to have successfully carried out, but then the orthoptic measures are not the incumbrance of this method alone, for if it is desired to obtain an ideal cure of strabismus, $i$. $e$, binocular single vision, it will be necessary to bring these measures into operation as a compliment to tenotomy.

In the clinic a good deal of difficulty is experienced in prevailing upon patients to strictly follow out the directions given for eye exercises; for this reason I have not laid so much stress on this part of the treatment in clinical practice. And in order to ascertain the results obtained by correction of the ametropia and the use of atropin alone, I began on the Ist of January, I 889 , to keep a private record of all the cases which applied for relief of this deformity in my service at the Illinois Charitable Eye and Ear Infirmary. The record is not as complete as might be desired, but it nevertheless shows in a conclusive manner what can be accomplished by the non-operative treatment.

The method pursued was as follows:

I. Measurement of angle of convergence, obtained with perimeter by Landolt's method. Examination of motility of squinting eye. Ophthalmoscopic measurement of refraction. Subjective examination of manifest hypermetropia. Test of vision. Instillation of atropin once or twice a day for a week, then measurement of angle of deviation. Measurement total hypermetropia.

2. Treatment: Glasses were prescribed correcting the total ametropia; atropin to be used daily until parallelism was obtained. Then gradual decrease of dose; until accommodation was performed without over-convergence. If after the effects of atropin had passed off, over- 
convergence reappeared, atropin was again ordered until desired result was obtained. The patient was then discharged with instructions to use the glasses constantly, and report if any tendency to squint should be noticed.

From January ist to June Ist, twenty-five consecutive cases are recorded. Of these there were of periodic strabismus 3, I cured, 2 much improved; I (angle of deviation decreased from $25^{\circ}$ to $5^{\circ}$ ). Alternating strabismus, I case, improved, axis nearly parallel. Established monocular strabismus convergens, 2 I cases. The right eye deviated in 15 , the left in 5 .

Results: In 10, parallelism. In one of these slight divergence was noted, due to operation 3 years ago ; 3 much improved, angle ranging from $5^{\circ}$ to $8^{\circ} ; 5$ not affected by treatment; 3 no record.

Parallelism was recorded in two or three cases, while still under atropin. Unsatisfactory as this may seem, it is so only in appearance, for if parallelism can be induced with atropin, it is only a question of time when it will continue without the mydriatic.

As children give very unsatisfactory answers when tested for glasses, they were prescribed by ophthalmoscopic measurement made under the effects of atropin. Measurements made before using atropin are unreliable. I may add that no difficulty was experienced in making the examinations and that the patients or their parents, were perfectly willing to procure the glasses and follow out the course of treatment. The details will be found noted in the tabulated record appended.

Without pretending to draw any positive conclusions or make sweeping generalizations from the twenty-five cases, the number being too small, I present them as illustrative of what the nonsurgical treatment.can accomplish even when deprived of that most valuable auxiliary, orthoptic exercises. To the fact that the record is not complete enough to satisfy the hypercritical, I am perfectly sensible, but the most important data will be found noted with as much accuracy as circumstances would permit. Out of twentyone cases of confirmed monocular strabismus convergens, ten were corrected, and three have been so much improved, that total correction is simply a question of time. Of three there is no record. Only five cases remained unaffected, and will be operated upon in the near future. Does not this record justify a thorough trial of the non-surgical method?

I beg you to believe, gentlemen, that I should not have presumed to present myself before you as an advocate of this method of treatment, if armed only with an experience derived from so scant a display of cases, but I am supported by a profound conviction of its value, the result of observations ranging back over nine years, during which I have given these cases considerable attention; and the motive which prompts this paper is not I assure you, a spirit of criticism, but an earnest desire to see a method which I know to be good, put more generally into practice. Careful observation and inquiry have elicited the information that hasty operative interference in concomitant strabismus convergens is unfortunately very common. Experience teaches that it is attended with danger. Is it not time to call a halt in our rush for radical measures, and consider carefully a method which commends itself, not only by its rationality, but, by its ability to correct in many instances this common deformity, and in others to prepare in the way for a safer and more accurate operative procedure.

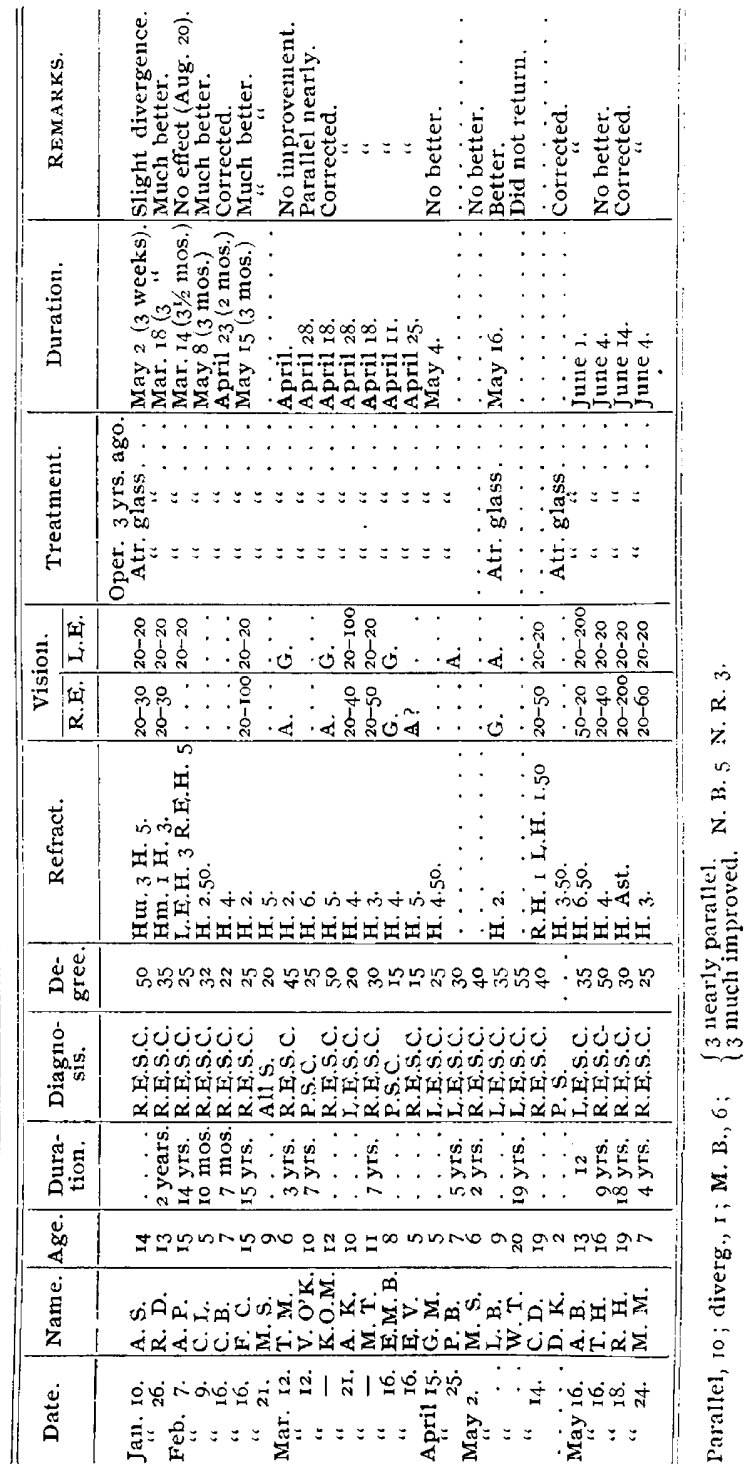

I 70 State Street.

DR. KNAPP said: For many years I have been 
in the habit of trying the non-surgical treatment in all young cases. A certain number have been cured, but the number was not so great as in Dr. G.'s series. It is a minority, perhaps not even what is called a respectable minority; certainly it does not reach 20 per cent. I have never operated before a fair trial with glasses has been made, for at least three months, mostly six months or a year or two. It seems that Dr. Gardiner has not drawn into the circle of his investigations the remaining latent convergence, which in my experience is constant or almost constant, which exists in most cases without asthenopic complaints.

DR. AYRFs, of Cincinnati, said that he considered the sentiment of the paper in the right direction. We all operate much less frequently than we did a few years ago. He had a case where glasses were worn for eight years, and the eyes were then parallel and there were no asthenopic symptoms. I encourage patients to use glasses, and try to keep them under observation for a year or two, if possible. I would hesitate to operate unless I could not see the effects of glasses for a period of a few months at least.

DR. KEYSER: I have been for some years carrying out the idea that Dr. Gardiner has presented, but I must suggest that a longer time be given before any operations are made. I agree with Dr. Knapp that it is necessary to wait a longer time than two or three weeks. It is necessary to wait three, four and six months. I have had some excellent results with the correction of the ametropia only after three to six months. The operation of tenotomy should not be made too soon after the correction. I always wait a considerable time, especially if I see any changes for the better are showing themselves. I lately exhibited to my class a boy with most perfect success of the correction of the strabismus with the correction of the ametropia, which took six months for the cure.

Dr. J. L. Thompson, of Indianapolis : Every year added to my experience makes me more cautious in recommending an operation for the cure of strabismus convergens, and causes me to urge the more frequent trial of the proper lenses instead. We should do all in our power to educate the people concerning this matter. Often have I prescribed glasses for children under 3 years of age and in one case before the second year, and in several this plan has sufficed without my hav. ing had to operate. Again, in cases where this has not been sufficient after six months, atropin has been used for three months in addition to the glasses, but the parents have also been urged to practice the defective eye many times a day. They must not simply be told that this is necessary, but they must also be informed that the state of the child's vision is almost altogether within their power. They must carry this injunction as did the Jews their laws, as frontlets between the eyes, written also on their gate posts, that, going in and out, to be read and practiced. Operations for strabismus are rarely even what we desire them to be. Perfect successes are seldom obtained, and in some, when the eye has become amblyopic from exclusion long before the operation, we may have an excellent immediate result from strabotomy, and this may continue for months or a year; and yet, in some of these, when they step into our offices, years afterwards, with their eyes turned out even more than they formerly turned in, such a terrible feeling of goneness comes over us that we wish they never had been touched. This must occur to all operators, no matter how skilful or expert they may be. If, then, perfect results rarely obtain from operative procedure in such cases, we should so educate the profession and, through them, the community, as to cause them to seek early advice, in order that benefit from glasses and other treatment may be entered upon.

DR. CHIsolm said that there was a general disposition to defer operation in crossed eyed children until they reach 8 years of age at least. We are frequently consulted by much younger children. During the delay or interval before the operation of tenotomy a good length of time admits of the use of glasses and atropin to allay excessive muscular spasm. For months this course is usually pursued, but my experience is that I do not get the large percentage of cures that others have obtained. I should think that Io per cent. of cures would be a larger percentage than my experience would warrant. Yet the method was a good one and is now generally adopted.

DR. STEVENS said: The view of procedure in strabismus will depend somewhat upon the view which we take of the nature of the cause of the defect. If we accept without question the theory of our great master, Donders, we shall act differently from what we would do were we to accept that of Schweigger and many others. If we are dealing with a simple functional trouble, we treat it as a functional trouble. If, however, we are dealing with anatomical conditions, we must deal with it by mechanical measures. There is a large proportion of cases of apparent convergent squint which, in my opinion, depend upon a vertical deviation. If we operate for convergent squint in such cases, we shall of course induce, at a later period, divergent strabismus. We can not, it appears to me, make sufficiently accurate estimations of the angle of strabismus by the perimeter. We should use the diplopia tests, and a full correcting operation should only be done by these tests.

In closing the discussion Dr. Gardiner stated that he had been misunderstood regarding the length of time he would follow up the treatment, the period of one month being the minimum limit in all cases. 